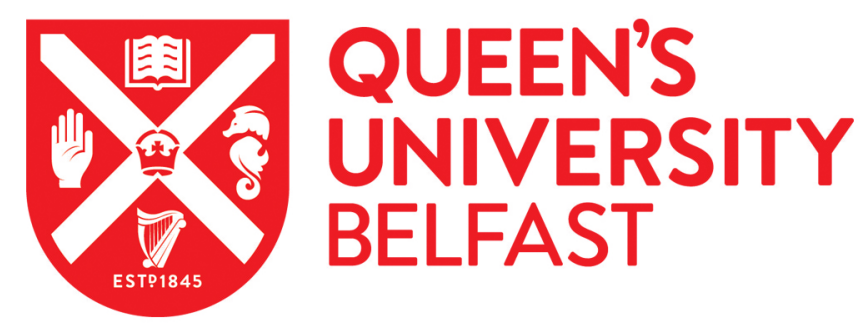

\title{
Improper Gaussian Signaling for Computationally Tractable Energy and Information Beamforming
}

Yu, H., Tuan, H. D., Nasir, A. A., Duong, T. Q., \& Hanzo, L. (2020). Improper Gaussian Signaling for Computationally Tractable Energy and Information Beamforming. IEEE Transactions on Vehicular Technology, 69(11), 13990 - 13995. https://doi.org/10.1109/TVT.2020.3025371

Published in:

IEEE Transactions on Vehicular Technology

Document Version:

Peer reviewed version

Queen's University Belfast - Research Portal:

Link to publication record in Queen's University Belfast Research Portal

Publisher rights

(c) 2020 IEEE.

This work is made available online in accordance with the publisher's policies. Please refer to any applicable terms of use of the publisher.

\section{General rights}

Copyright for the publications made accessible via the Queen's University Belfast Research Portal is retained by the author(s) and / or other copyright owners and it is a condition of accessing these publications that users recognise and abide by the legal requirements associated with these rights.

Take down policy

The Research Portal is Queen's institutional repository that provides access to Queen's research output. Every effort has been made to ensure that content in the Research Portal does not infringe any person's rights, or applicable UK laws. If you discover content in the Research Portal that you believe breaches copyright or violates any law, please contact openaccess@qub.ac.uk. 


\title{
Improper Gaussian Signaling for Computationally Tractable Energy and Information Beamforming
}

\author{
H. Yu ${ }^{1,2}$, H. D. Tuan ${ }^{2}$, A. A. Nasir ${ }^{3}$, T. Q. Duong ${ }^{4}$ and L. Hanzo ${ }^{5}$
}

\begin{abstract}
The transmit time-switching (transmit-TS) approach, under which the energy and information are transferred over different fractions of a time slot has proved its supremacy over the power splitting (PS) approach of simultaneous wireless information and power transfer, where PS splits the power of the received signal for energy harvesting and information decoding. For integrating data and energy transfer, this paper develops new classes of beamforming that are suitable for improper Gaussian signaling which is capable of network throughput improvements while maintaining high computational efficiency in its design.
\end{abstract}

Index Terms-Improper Gaussian signaling, multi-user interference system, energy-harvesting, nonconvex optimization, qualityof-service (QoS).

\section{INTRODUCTION}

Modern wireless networks aim to deliver both information and energy over wireless channels [1]-[3]. Recent studies have shown [4], [5] that the transmit time-switching (transmit-TS), which transmits power within a fraction of the time slot (TS) and then transmits information within the remaining fraction, is capable of outperforming the power splitting (PS) approach of simultaneous wireless information and power transfer (SWIPT), where PS splits the power of the received signal for energyharvesting (EH) and information detection (ID). In contrast to PS, which relies on a joint data and energy beamformer, transmit-TS benefits from having a distinct energy transmit beamformer (ETBF) for power delivery and information transmit beamformer (ITBF) for information delivery and thus is very efficient. More particularly, it has been shown in [6] that under the same quality-of-service (QoS) for harvested energy, transmit-TS relying on conjugate beamforming $(\mathrm{CBF})$ for $\mathrm{EH}$ and zero-forcing beamforming (ZFBF) for ID, substantially outperforms the SWIPT technique of [7] in terms of the users' max-min information throughput. ZFBF is applicable when the number of users served is much lower than the number of transmit antennas (TAs) so the right inverse of the channel matrix is well conditioned. As the number of users increases, forcing the multi-user interference to zero becomes impossible because instead of simple ZFB, one has to employ

\footnotetext{
${ }^{1}$ School of Communication and Information Engineering, Shanghai University, Shanghai, China (email: hongwenyu@ shu.edu.cn); ${ }^{2}$ School of Electrical and Data Engineering, University of Technology, Sydney, NSW 2007, Australia (email: hongwen.yu@uts.edu.au, tuan.hoang@uts.edu.au); ${ }^{3}$ Department of Electrical Engineering, King Fahd University of Petroleum and Minerals (KFUPM), Dhahran, Saudi Arabia (e-mail: anasir@kfupm.edu.sa); ${ }^{4}$ Queen’s University Belfast, Belfast BT7 1NN, UK (email: trung.q.duong@qub.ac.uk); ${ }^{5}$ School of Electronics and Computer Science, University of Southampton, Southampton, SO17 1BJ, U.K. (e-mail: 1h@ecs.soton.ac.uk)

$\mathrm{H}$. Yu would like to acknowledge the financial support of the National Natural Science Foundation of China under Grant 61901254.

H. D. Tuan would like to acknowledge the financial support of the Institute for Computational Science and Technology, Hochiminh City, Vietnam and the Australian Research Council's Discovery Projects under Grant DP190102501.

L. Hanzo would like to acknowledge the financial support of the Engineering and Physical Sciences Research Council projects EP/N004558/1, EP/P034284/1, EP/P034284/1, EP/P003990/1 (COALESCE), of the Royal Society's Global Challenges Research Fund Grant as well as of the European Research Council's Advanced Fellow Grant QuantCom.
}

other computationally tractable beamforming classes such as regularized ZFBF (RZF-BF) [8], which regularizes the channel matrix for the right inversion, or generalized ZFBF (GZF$\mathrm{BF}$ ) [9], which nullifies the approximated interfering matrices. However, proper Gaussian signaling (PGS) is only optimal in interference-free systems [10]. As a further development, we have shown in [11] that improper Gaussian signaling (IGS) (see e.g. [12], [13] and references therein) is capable of substantially improving the information throughput of energy-harvesting aided networks. However, a beamforming design challenge of IGS is its computational tractability, since it poses largedimensional nonconvex problems, which involve $N_{t}(K+2 M)$ complex decision variables for scenarios of an $N_{t}$ antenna base station (BS) serving $K \mathrm{EH}$ users (EUs) and $M$ informationreceiving users (IUs). IGS is conventionally based on widely linear beamforming, which consists of a pair of beamformers, namely one for proper Gaussian sources and one for their conjugate. Hence, the RZF-BF and GZF-BF schemes used in PGS cannot be readily extended to the associate widely linear beamforming. By contrast, based on linear beamforming of improper Gaussian sources, this paper develops a new class of RZF-BF and GZF-BF that is eminently suitable for IGS, which involves only $K+M$ real decision variables and $M$ complex decision variables for computation. A byproduct is thus RZF$\mathrm{BF}$ and GZF-BF under IGS for information transmission, which cannot be found in the existing literature. To sum up, we provide a brief comparison of the related literature in Table I. The problem of the information users' max-min throughput

TABLE I: A brief comparison of the related literature.

\begin{tabular}{|l|c|c|c|c|}
\hline Contents & This work & [11] & [4] & [7] \\
\hline transmit TS & $\sqrt{ }$ & $\sqrt{ }$ & $\sqrt{ }$ & \\
\hline SWIPT & $\sqrt{ }$ & & $\sqrt{ }$ & $\sqrt{ }$ \\
\hline PGS & & $\sqrt{ }$ & $\sqrt{ }$ & $\sqrt{ }$ \\
\hline IGS & $\sqrt{ }$ & $\sqrt{ }$ & & \\
\hline CBF only & & & & $\sqrt{ }$ \\
\hline RZF-BF & $\sqrt{ }$ & & & \\
\hline GZF-BF & $\sqrt{ }$ & & & \\
\hline
\end{tabular}

optimization subject to the EH constraints of energy users under the transmit-TS approach is addressed in Section II and Section III. Section IV also addresses a similar problem, but under the PS regime to show that IGS also helps improve the ID performance in SWIPT. The simulations provided in Section V confirm the advantage of the transmit-TS over PS under IGS, and the advantage of IGS over PGS under SWIPT. Section VI concludes the paper.

Notation. Bold-face lower-case and upper-case letters are respectively used for vectors and matrices; $[\mathbf{X}]^{2}$ is $\mathbf{X X}{ }^{H}$, and $\langle\mathbf{X}, \mathbf{Y}\rangle=\triangleq \operatorname{trace}\left(\mathbf{X}^{H} \mathbf{Y}\right)$; we also use $\langle\mathbf{A}\rangle \triangleq \operatorname{trace}(\mathbf{A})$; for a set of subscription indexes $\mathcal{S}, x_{\mathcal{S}} \triangleq\left\{x_{s}: s \in \mathcal{S}\right\}$ and $x_{\mathcal{S}}>0$ means that $x_{s}>0 \forall s \in \mathcal{S}$, while $\{\mathcal{A}\}>0$ means $a>0 \forall a \in \mathcal{A} ; \mathbf{I}_{n}$ is the identity matrix of size 
$n \times n ; \operatorname{diag}\left[a_{1}, a_{2}\right]$ is a diagonal matrix of size $2 \times 2$ with the diagonal entries $a_{1}$ and $a_{2}$. Col $\left[\mathbf{h}_{i}\right]_{i=1}^{M}$ arranges the vectors $\mathbf{h}_{i}, i=1, \ldots, M$ into a single (column) vector. The following inequalities are frequently used in the paper for all matrices $\mathbf{V}$, positive semi-definite matrices $\mathbf{X}$ and $\overline{\mathbf{X}}$ and matrix $\overline{\mathbf{V}}$, positive definite matrices $\mathbf{Y}$ and $\overline{\mathbf{Y}}$ of size $n \times n$ [14], [15]:

$$
\begin{array}{r}
\ln \left|\mathbf{I}_{n}+\mathbf{X} \mathbf{Y}^{-1}\right| \geq \ln \left|\mathbf{I}_{n}+\overline{\mathbf{X}} \overline{\mathbf{Y}}^{-1}\right|+2 n-\langle\overline{\mathbf{Y}}-1, \mathbf{Y}\rangle \\
-\left\langle\overline{\mathbf{X}}+\overline{\mathbf{Y}},(\mathbf{X}+\mathbf{Y})^{-1}\right\rangle, \\
\ln \left|\mathbf{I}_{n}+[\mathbf{V}]^{2} \mathbf{Y}^{-1}\right| \geq \ln \left|\mathbf{I}_{n}+[\overline{\mathbf{V}}]^{2} \overline{\mathbf{Y}}^{-1}\right|-\left\langle[\overline{\mathbf{V}}]^{2} \overline{\mathbf{Y}}^{-1}\right\rangle \\
+2 \Re\left\{\left\langle\overline{\mathbf{V}}^{H} \overline{\mathbf{Y}}^{-1} \mathbf{V}\right\rangle-\left\langle\overline{\mathbf{Y}}^{-1}-\left([\overline{\mathbf{V}}]^{2}+\overline{\mathbf{Y}}\right)^{-1},[\mathbf{V}]^{2}+\mathbf{Y}\right\rangle .\right.
\end{array}
$$

\section{RZF-BF UNDER IGS}

Consider the downlink of a $N_{t}$-antenna aided BS serving $K$ EUs indexed by $e_{k}, k \in \mathcal{K} \triangleq\{1, \ldots, K\}$, which have to be located sufficiently near to the BS, and $M$ IUs indexed by $d_{m}$, $m \in \mathcal{M}=\{1, \ldots, M\}$. There is a potential overlap between the sets of EUs and IUs namely for users, who act as both EUs and IUs. All users are equipped with a single antenna.

Under transmit-TS, a fraction of time $0<1 / t_{1}<1$ is used for power transfer, while the remaining fraction of time $0<1 / t_{2}<1$ is used for information transfer. Let $\mathbf{h}_{e_{j}} \in \mathbb{C}^{1 \times N_{t}}$ be the channel spanning from the BS to EU $e_{j}$, and $s_{e_{j}}$ be the energy symbol with $\left|s_{e_{j}}\right|^{2}=1$. By using CBF $\sum_{k=1}^{K} \sqrt{\tilde{p}_{k}} \mathbf{h}_{e_{k}}^{H} s_{e_{k}}$ for energy transfer, so $\tilde{p}_{\mathcal{K}}$ represent the power allocation, the energy harvested by EU $e_{j}$ is $\left(1 / t_{1}\right) \zeta\left\|\mathbf{y}_{e_{j}}\right\|^{2}=\left(1 / t_{1}\right) \zeta \pi_{j}\left(\tilde{p}_{\mathcal{K}}\right)$, where

$$
\pi_{j}\left(\tilde{p}_{\mathcal{K}}\right)=\sum_{k=1}^{K}\left|\mathbf{h}_{e_{j}} \mathbf{h}_{e_{k}}^{H}\right|^{2} \tilde{p}_{k}, j \in \mathcal{K},
$$

and $0<\zeta<1$ is the efficiency of energy conversion at the $\mathrm{EH}$ receiver, which is set to the typical value of 0.5 in the paper.

Let $\mathbf{h}_{d_{\ell}} \in \mathbb{C}^{1 \times N_{t}}$ be the channel from the BS to IU $d_{\ell}$. For information transfer during the remaining $1 / t_{2}$ fraction of time, the transmit signal for IU $d_{m}$ termed as $\mathbf{x}_{d_{m}}$ is generated from an improper Gaussian source $s_{d_{m}}$ as

$$
\mathbf{x}_{d_{m}}=\mathbf{f}_{m} s_{d_{m}}
$$

where $\mathbf{f}_{m} \in \mathbb{C}^{N_{t}}$ is a pre-defined vector, with $\left\|\mathbf{f}_{m}\right\|=1$.

Define $\sqrt{\beta_{\ell}} \triangleq\left\|\mathbf{h}_{d_{\ell}}\right\|, \tilde{\mathbf{h}}_{d_{\ell}}=\mathbf{h}_{d_{\ell}} /\left\|\mathbf{h}_{d_{\ell}}\right\|, \mathbf{H} \triangleq \operatorname{Col}\left[\mathbf{h}_{d_{i}}\right]_{i=1}^{M}$, and $\tilde{\mathbf{H}} \triangleq \operatorname{Col}\left[\tilde{\mathbf{h}}_{d_{i}}\right]_{i=1}^{M}$. We will use the following innovative class of RZF-BF proposed in [14]:

$$
\left[\begin{array}{lll}
\mathbf{f}_{1} & \ldots & \mathbf{f}_{M}
\end{array}\right]=\tilde{\mathbf{H}}^{H}\left(\tilde{\mathbf{H}} \tilde{\mathbf{H}}^{H}+\alpha \mathbf{I}_{M}\right)^{-1},
$$

which regularizes only the ill-conditioned part $\tilde{\mathbf{H}}$ of the channel matrix $\mathbf{H}$, instead of the conventional RZF-BF [8] defined by

$$
\left[\begin{array}{lll}
\mathbf{f}_{1} & \ldots & \mathbf{f}_{M}
\end{array}\right]=\mathbf{H}\left(\mathbf{H} \mathbf{H}^{H}+\alpha \mathbf{I}_{M}\right)^{-1},
$$

which regularizes the whole channel matrix $\mathbf{H}$.

The signal received at $\mathrm{IU} d_{\ell}$ is

$$
y_{d_{\ell}}=\mathbf{h}_{d_{\ell}} \sum_{m=1}^{M} \mathbf{f}_{m} s_{d_{m}}+n_{d_{\ell}}, \ell \in \mathcal{M},
$$

where $n_{d_{\ell}} \sim \mathcal{C} \mathcal{N}(0, \sigma)$ is the background noise.
For $p_{\ell}=\mathbb{E}\left(\left|s_{d_{\ell}}\right|^{2}\right)$ and $q_{\ell}=\mathbb{E}\left(\left(s_{d_{\ell}}\right)^{2}\right)$, the augmented covariance of $s_{d_{\ell}}$ is defined by

$$
\mathbf{C}\left(p_{\ell}, q_{\ell}\right) \triangleq\left[\begin{array}{ll}
p_{\ell} & q_{\ell} \\
q_{\ell}^{*} & p_{\ell}
\end{array}\right] .
$$

For $\mathbf{G}_{\ell, m}=\operatorname{diag}\left[\mathbf{h}_{\ell} \mathbf{f}_{m},\left(\mathbf{h}_{\ell} \mathbf{f}_{m}\right)^{*}\right]$, the information throughput at UE $d_{\ell}$ is $\left(1 / 2 t_{2}\right) r_{\ell}\left(p_{\mathcal{M}}, q_{\mathcal{M}}\right)$ with [16]

$$
r_{\ell}\left(p_{\mathcal{M}}, q_{\mathcal{M}}\right)=\ln \left|\mathbf{I}_{2}+\mathbf{G}_{\ell, \ell} \mathbf{C}\left(p_{\ell}, q_{\ell}\right) \mathbf{G}_{\ell, \ell}^{H} \mathcal{L}_{\ell}^{-1}\left(p_{\mathcal{M}}, q_{\mathcal{M}}\right)\right|
$$

for $\mathcal{L}_{\ell}\left(p_{\mathcal{M}}, q_{\mathcal{M}}\right) \triangleq \sum_{m \in \mathcal{M} \backslash\{\ell\}} \mathbf{G}_{\ell, m} \mathbf{C}\left(p_{m}, q_{m}\right) \mathbf{G}_{\ell, m}^{H}+\sigma \mathbf{I}_{2}$.

It is straightforward to show that when all information sources $s_{d_{m}}$ are proper Gaussian $\left(q_{\ell} \equiv 0\right)$ :

$$
r_{\ell}\left(p_{\mathcal{M}}, 0\right)=2 \ln \left(1+\frac{\left|\mathbf{h}_{\ell} \mathbf{f}_{\ell}\right|^{2} p_{\ell}}{\sum_{m \in \mathcal{M} \backslash\{\ell\}}\left|\mathbf{h}_{\ell} \mathbf{f}_{m}\right|^{2} p_{m}+\sigma}\right)
$$

and the information throughput at $\mathrm{UE} d_{\ell}$ is the conventional throughput $\left(1 / t_{2}\right) r_{\ell}\left(p_{\mathcal{M}}, 0\right)$.

For the linear functions $\pi_{1}\left(\tilde{p}_{\mathcal{K}}\right) \triangleq \sum_{j=1}^{K}\left\|h_{e_{j}}\right\|^{2} \tilde{p}_{j}$ and $\pi_{2}\left(p_{\mathcal{M}}\right) \triangleq \sum_{m=1}^{M}\left\|\mathbf{f}_{m}\right\|^{2} p_{m}$, we aim for solving the following problem of max-min throughput optimization:

$$
\begin{array}{r}
\max _{\left\{\tilde{p}_{\mathcal{K}}>0, p_{\mathcal{M}}>0, t_{1}, t_{2}\right\}>0, q_{\mathcal{M}}, \gamma} \gamma \\
\text { s.t. } \quad \pi_{j}\left(\tilde{\mathbf{p}}_{\mathcal{K}}\right) \geq e_{\min } t_{1} / \zeta, j \in \mathcal{K}, \\
r_{\ell}\left(p_{\mathcal{M}}, q_{\mathcal{M}}\right) \geq 2 \gamma t_{2}, \ell \in \mathcal{M}, \\
1 / t_{1}+1 / t_{2} \leq 1, \\
\left|q_{m}\right| \leq p_{m}, m \in \mathcal{M}, \\
\left\|h_{e_{j}}\right\|^{2} \tilde{p}_{j} \leq P, j \in \mathcal{K} ;\left\|f_{m}\right\|_{F}^{2} p_{m} \leq P, m \in \mathcal{M}, \\
\pi_{1}\left(\tilde{\mathbf{p}}_{\mathcal{K}}\right) / t_{1}+\pi_{2}\left(\mathbf{p}_{\mathcal{M}}\right) / t_{2} \leq P,
\end{array}
$$

where (9b) is the energy constraint of EUs in terms of their minimum required energy, the slack variable $\gamma$ is introduced in (9c) to express the IUs minimum throughput; (9d) restricts the energy and information transfer within a time slot; the constraint (9e) guarantees that $\mathbf{C}\left(p_{\ell}, q_{\ell}\right)$ is qualified to be the augmented covariance of an improper Gaussian source with power $p_{m}$; (9f) is the total power transmit constraint under a given budget $P$, and $(9 \mathrm{~g})$ is a physical transmission constraint. Also, $\alpha=M \sigma / P$ in (5) is set. This problem is nonconvex because the two constraints (9c) and (9f) are nonconvex: the left hand side (LHS) of (9c) is a nonconcave function, while its right hand side (RHS) is a nonconvex function, and both terms in (9f) are nonconvex functions (since they are linear fractional functions). To propose a path-following algorithm for computing (9), we have to develop inner approximations for these constraints.

Let $\left(\tilde{p}_{\mathcal{K}}^{(\kappa)}, p_{\mathcal{M}}^{(\kappa)}, q_{\mathcal{M}}^{(\kappa)}, t_{1}^{(\kappa)}, t_{2}^{(\kappa)}, \gamma^{(\kappa)}\right)$ be the feasible point for (9) found from the $(\kappa-1)$-th iteration. By using the inequality (1), we have

$$
r_{\ell}\left(p_{\mathcal{M}}, q_{\mathcal{M}}\right) \geq r_{\ell}^{(\kappa)}\left(p_{\mathcal{M}}, q_{\mathcal{M}}\right),
$$

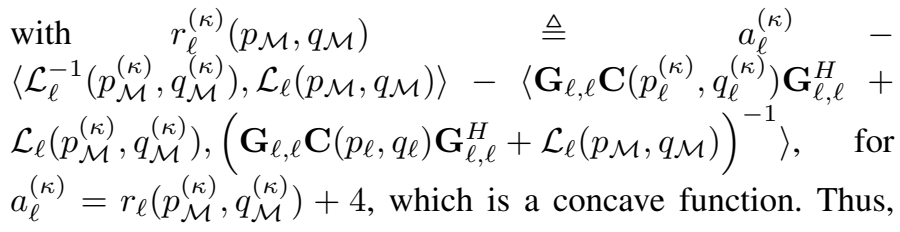




\section{Algorithm 1 Path-following algorithm for computing (9)}

1: Initialization: Fix some $\left(t_{1}^{(0)}, t_{2}^{(0)}\right)$ and good $\gamma^{(0)}$ to randomly generate a feasible point $\left(\tilde{p}^{(0)}, p_{\mathcal{K}}^{(0)}, q_{\mathcal{M}}^{(0)}, t_{1}^{(0)}, t_{2}^{(0)}, \gamma^{(0)}\right)$ for the convex constraints (9b), (9e), (9f), (9g). Iterate $\max _{\left(\tilde{p}_{\mathcal{K}}, p_{\mathcal{M}}, \eta\right)>0, q_{\mathcal{M}}} \eta$ s.t. (9b), (9e), (9f), (9g), $r_{\ell}^{(\kappa)}\left(p_{\mathcal{M}}, q_{\mathcal{M}}\right) \geq 2 \gamma^{(0)} t_{2}^{(0)} \eta$ until reaching $\eta \geq 1$. Reset $\left(\tilde{p}_{\mathcal{K}}^{(0)}, p_{\mathcal{M}}^{(0)}, q_{\mathcal{M}}^{(0)}, t_{1}^{(0)}, t_{2}^{(0)}, \gamma^{(0)}\right) \leftarrow$ $\left(\tilde{p}_{\mathcal{K}}^{(\kappa)}, p_{\mathcal{M}}^{(\kappa)}, q_{\mathcal{M}}^{(\kappa)}, t_{1}^{(0)}, t_{2}^{(0)}, \gamma^{(0)}\right)$ as a feasible point for (9).

Set $\kappa=0$.

2: Repeat until convergence of the objective function in (9a): Solve the convex problem (13) to generate $\left(\tilde{p}_{\mathcal{K}}^{(\kappa+1)}, p_{\mathcal{M}}^{(\kappa+1)}, p_{\mathcal{M}}^{(\kappa+1)}, t_{1}^{(\kappa+1)}, t_{2}^{(\kappa+1)}, \gamma^{(\kappa+1)}\right)$ for (9). Set $\kappa \leftarrow \kappa+1$.

3: Output $\left(\tilde{p}_{\mathcal{K}}^{(\kappa)}, p_{\mathcal{M}}^{(\kappa)}, p_{\mathcal{M}}^{(\kappa)}, t_{1}^{(\kappa)}, t_{2}^{(\kappa)}, \gamma^{(\kappa)}\right)$ and accept it as the optimal solution for (9).

the nonconvex constraint (9c) is innerly approximated [17] by the following convex constraint ${ }^{1}$

$$
r_{\ell}^{(\kappa)}\left(p_{\mathcal{M}}, q_{\mathcal{M}}\right) \geq \gamma^{(\kappa)} t_{2}^{(\kappa)}\left(\gamma / \gamma^{(\kappa)}+t_{2} / t_{2}^{(\kappa)}\right)^{2}, \ell \in \mathcal{M},
$$

where its RHS, which is a convex quadratic function, is an upper approximation [17] of the nonconvex function on the RHS of (9c).

Furthermore, the nonconvex constraint (9f) is innerly approximated by the following convex constraint

$$
\frac{\pi_{1}\left(\tilde{p}_{\mathcal{K}}^{(\kappa)}\right) t_{2}^{(\kappa)}}{4 t_{1}}\left(\frac{\pi_{1}\left(\tilde{p}_{\mathcal{K}}\right)}{\pi_{1}\left(\tilde{p}_{\mathcal{K}}^{(\kappa)}\right)}+\frac{t_{2}}{t_{2}^{(\kappa)}}\right)^{2}+\pi_{2}\left(p_{\mathcal{M}}\right) \leq P t_{2} .
$$

At the $\kappa$-th iteration, we solve the following convex problem to generate the next feasible point $\left(\tilde{p}_{\mathcal{K}}^{(\kappa+1)}, p_{\mathcal{M}}^{(\kappa+1)}, q_{\mathcal{M}}^{(\kappa)}, t_{1}^{(\kappa+1)}, t_{2}^{(\kappa+1)}, \gamma^{(\kappa+1)}\right)$ for $(9)$ :

$\max _{\left\{\tilde{p}_{\mathcal{K}}, p_{\mathcal{M}}, t_{1}, t_{2}, \gamma\right\}>0, q_{\mathcal{M}}}$

$$
\gamma \text { s.t. }(9 b),(9 d),(9 e),(9 g),(11),(12) \text {. (13) }
$$

Note that $\gamma^{(\kappa+1)}$ and $\gamma^{(\kappa)}$ are the optimal value and a feasible value for (13) respectively, hence we have $\gamma^{(\kappa+1)}>\gamma^{(\kappa)}$ as far as $\gamma^{(\kappa+1)} \neq \gamma^{(\kappa)}$. As such, the sequence $\left\{\left(\tilde{p}_{\mathcal{K}}^{(\kappa)}, p_{\mathcal{M}}^{(\kappa)}, q_{\mathcal{M}}^{(\kappa)}, t_{1}^{(\kappa)}, t_{2}^{(\kappa)}, \gamma^{(\kappa)}\right)\right\}$ is of improved feasible points for the nonconvex problem (9) converges at least to a locally optimal solution of (9) [4]. Algorithm 1 provides the pseudo-code for implementing the proposed path-following computational procedure.

\section{GZF-BF UNDER IGS}

For each $m \in \mathcal{M}$, the matrix $\mathbf{H}_{m}$ of size $(M-1) \times N_{t}$ obtained by excluding $\mathbf{h}_{d_{m}}$ from the matrix $\mathbf{H}$ of size $M \times N_{t}$ expresses the interference of the transmit signal for IU $d_{m}$ to other IUs. We employ the singular value decomposition (SVD)

$$
\mathbf{H}_{m}=\mathbf{U}_{m} \Sigma_{m} \mathbf{V}_{m},
$$

where $\mathbf{U}_{m}$ is an orthogonal matrix $\left(\mathbf{U}_{m}^{H} \mathbf{U}_{m}=\mathbf{I}_{N_{t}}\right)$ of size $(M-1) \times N_{t}, \Sigma_{m}$ is a diagonal matrix of size $(M-1) \times N_{t}$,

\footnotetext{
${ }^{1}(11)$ is an inner convex approximation of the nonconvex constraint $(9 \mathrm{c})$ in the sense that each feasible point for the former is also feasible for the latter and provides a lower bound for the optimal value of the former.
}

which contains the singular values $\sigma_{m, \ell}, \ell=1, \ldots, N_{t}$ of $\mathbf{H}_{m}$ in decreasing order on its diagonal, and $\mathbf{V}_{m}$ is an unitary matrix of size $N_{t} \times N_{t}$.

Let $\eta_{m} \triangleq \operatorname{rank}\left(\mathbf{H}_{m}\right)$, and $\iota_{m} \triangleq \max \left\{2, \eta_{m} / 3\right\}$. By keeping only the largest $\eta_{m}-\iota_{m}$ singular values and setting all other singular values on the diagonal of $\Sigma_{m}$ to zero to obtain $\hat{\Sigma}_{m}$, we define

$$
\hat{\mathbf{H}}_{m} \triangleq \mathbf{U}_{m} \hat{\Sigma}_{m} \mathbf{V}_{m},
$$

which is the best rank $\left(\eta_{m}-\iota_{m}\right)$ approximation of $\mathbf{H}_{m}$.

Let $\boldsymbol{\xi}_{m, i} \in \mathbb{C}^{N_{t}}, i=1, \ldots, \iota_{m}$ be the base of the null space of $\hat{\mathbf{H}}_{m}$. Instead of RZF-BF defined from (5), for $\alpha_{m} \triangleq\left\{\alpha_{m, i}, i=1, \ldots, \iota_{m}\right\}$, we seek the beamformer $\mathbf{f}_{m}$ in (4) in the form

$$
\mathbf{f}_{m}\left(\alpha_{m}\right)=\sum_{i=1}^{\iota_{m}} \alpha_{m, i} \boldsymbol{\xi}_{m, i}, \alpha_{m, i} \in \mathbb{C} .
$$

Like (8), for $\alpha \triangleq\left\{\alpha_{m}, m \in \mathcal{M}\right\}$, the information throughput at $\mathrm{UE} d_{\ell}$ is $\left(1 / 2 t_{2}\right) \hat{r}_{\ell}\left(\alpha, p_{\mathcal{M}}, q_{\mathcal{M}}\right)$ with

$$
\begin{array}{r}
\hat{r}_{\ell}\left(\alpha, p_{\mathcal{M}}, q_{\mathcal{M}}\right)=\ln \mid \mathbf{I}_{2}+\mathbf{G}_{\ell, \ell}(\alpha) \mathbf{C}\left(p_{\ell}, q_{\ell}\right) \mathbf{G}_{\ell, \ell}^{H}(\alpha) \\
\times \Psi_{\ell}^{-1}\left(\alpha, p_{\mathcal{M}}, q_{\mathcal{M}}\right) \mid
\end{array}
$$

where $\mathbf{G}_{\ell, m}(\alpha) \triangleq \operatorname{diag}\left[\mathbf{h}_{\ell} \mathbf{f}_{m}\left(\alpha_{m}\right),\left(\mathbf{h}_{\ell} \mathbf{f}_{m}\left(\alpha_{m}\right)\right)^{*}\right]$, and

$$
\Psi_{\ell}\left(\alpha, p_{\mathcal{M}}, q_{\mathcal{M}}\right) \triangleq \sum_{m \in \mathcal{M} \backslash\{\ell\}}\left[\mathbf{G}_{\ell, m}(\alpha) \sqrt{\mathbf{C}\left(p_{m}, q_{m}\right)}\right]^{2}+\sigma \mathbf{I}_{2} .
$$

Similarly to (9), the problem of max-min throughput optimization is formulated by

$$
\begin{array}{r}
\max _{\left\{\tilde{p}_{\mathcal{K}}, p_{\mathcal{M}}, t_{1}, t_{2}, \gamma\right\}>0, q_{\mathcal{M}, \alpha}} \gamma \quad \text { s.t. } \quad(9 b),(9 d)-(9 g), \\
\hat{r}_{\ell}\left(\alpha, p_{\mathcal{M}}, q_{\mathcal{M}}\right) \geq 2 \gamma t_{2}, \ell \in \mathcal{M}, \\
\sum_{i=1}^{\iota_{m}}\left|\alpha_{m, i}\right|^{2} \leq 1, m \in \mathcal{M},
\end{array}
$$

where (16c) guarantees that $\left\|\mathbf{f}_{m}\left(\alpha_{m}\right)\right\| \leq 1, m \in \mathcal{M}$.

For $\mathbf{f}_{m}=\mathbf{f}_{m}\left(\alpha_{m}^{(0)}\right)$ with $\alpha_{m}^{(0)} \triangleq\left\{\alpha_{m, i}^{(0)}, i=1, \ldots, i_{m}\right\}$ and $\alpha_{m, i}^{(0)} \triangleq e^{-\jmath \angle\left(\mathbf{h}_{d_{m}} \boldsymbol{\xi}_{m, i}\right)}\left|\mathbf{h}_{d_{m}} \boldsymbol{\xi}_{m, i}\right| /\left(\sum_{i=1}^{\iota_{m}}\left|\mathbf{h}_{d_{m}} \boldsymbol{\xi}_{m, i}\right|^{2}\right)^{1 / 2}$, we solve the convex problem (13) to generate $\left(\tilde{p}_{\mathcal{K}}^{(0)}, p_{\mathcal{M}}^{(0)}, p_{\mathcal{M}}^{(0)}, t_{1}^{(0)}, t_{2}^{(0)}, \gamma^{(0)}\right)$.

At every $\kappa$-th iteration, we process the following alternating optimization steps:

1) Alternating optimization of the normalized beamformer weights $\alpha_{m}$ : For $\alpha_{m}^{(\kappa)} \triangleq\left\{\alpha_{m, i}^{(\kappa)}, i=1, \ldots, \iota_{m}\right\}$ and $\alpha^{(\kappa)} \triangleq$ $\left\{\alpha_{m}^{(\kappa)}, m \in \mathcal{M}\right\}$, we use the inequality (2) to obtain

$$
\hat{r}_{\ell}\left(\alpha, p_{\mathcal{M}}^{(\kappa)}, q_{\mathcal{M}}^{(\kappa)}\right) \geq \hat{r}_{\ell}^{(\kappa)}(\alpha)
$$

with the concave function $\hat{r}_{\ell}^{(\kappa)}$ defined by $\hat{r}_{\ell}^{(\kappa)}(\alpha) \triangleq \tilde{a}_{\ell}^{(\kappa)}+$ $2 \Re\left\{\left\langle\mathbf{C}\left(p_{\ell}^{(\kappa)}, q_{\ell}^{(\kappa)}\right) \mathbf{G}_{\ell, \ell}^{H}\left(\alpha^{(\kappa)}\right) \Psi_{\ell}^{-1}\left(\alpha^{(\kappa)}, p_{\mathcal{M}}^{(\kappa)}, q_{\mathcal{M}}^{(\kappa)}\right) \mathbf{G}_{\ell, \ell}(\alpha)\right\rangle\right\}-$ $\left\langle\Psi_{\ell}^{-1}\left(\alpha^{(\kappa)}, p_{\mathcal{M}}^{(\kappa)}, q_{\mathcal{M}}^{(\kappa)}\right)-\left(\Psi_{\ell}\left(\alpha^{(\kappa)}, p_{\mathcal{M}}^{(\kappa)}, q_{\mathcal{M}}^{(\kappa)}\right)+\left[\mathbf{G}_{\ell, \ell}\left(\alpha^{(\kappa)}\right)\right.\right.\right.$

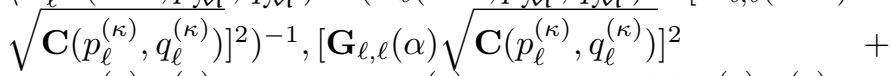
$\left.\left.\Psi\left(\alpha, p_{\mathcal{M}}^{(\kappa)}, q_{\mathcal{M}}^{(\kappa)}\right)\right)\right\rangle$, where $\tilde{a}_{\ell}^{(\kappa)} \triangleq \hat{r}_{\ell}\left(\alpha^{(\kappa)}, p_{\mathcal{M}}^{(\kappa)}, q_{\mathcal{M}}^{(\kappa)}\right)-$ $\left\langle\left[G_{\ell, \ell}\left(\alpha^{(\kappa)}\right) \sqrt{C_{\ell}^{A,(\kappa)}}\right]^{2} \Psi_{\ell}^{-1}\left(\alpha^{(\kappa)}, p_{\mathcal{M}}^{(\kappa)}, q_{\mathcal{M}}^{(\kappa)}\right)\right\rangle$.

We then solve the following problem to generate a better $\alpha^{(\kappa+1)}$ :

$$
\max _{\alpha} \min _{\ell=1, \ldots, M} \tilde{\rho}_{\ell}^{(\kappa)}(\alpha) \quad \text { s.t. } \quad(16 c) .
$$


2) Alternating power optimization of improper Gaussian sources: With $\mathbf{f}_{m}=\mathbf{f}_{m}\left(\alpha_{m}^{(\kappa+1)}\right)$, we solve the convex problem (13) to generate a better $\left(\tilde{p}_{\mathcal{K}}^{(\kappa+1)}, p_{\mathcal{M}}^{(\kappa+1)}, q_{\mathcal{M}}^{(\kappa+1)}, t_{1}^{(\kappa+1)}, t_{2}^{(\kappa+1)}, \gamma^{(\kappa+1)}\right)$.

The above steps generate a sequence $\left\{\left\{\mathbf{f}_{m}\left(\alpha_{m}^{(\kappa)}\right), m \in\right.\right.$ $\left.\mathcal{M}\}, \tilde{p}_{\mathcal{K}}^{(\kappa)}, p_{\mathcal{M}}^{(\kappa)}, q_{\mathcal{M}}^{(\kappa)}, t_{1}^{(\kappa)}, t_{2}^{(\kappa)}, \gamma^{(\kappa)}\right\} \quad$ of improved feasible points for (16), which converges to a feasible point satisfying the Karush-Kuhn-Tucker (KKT) condition in $\alpha$ (in $\left(\tilde{p}_{\mathcal{K}}, p_{\mathcal{M}}, q_{\mathcal{M}}, t_{1}, t_{2}, \gamma\right)$, resp.) with $\left(\tilde{p}_{\mathcal{K}}, p_{\mathcal{M}}, q_{\mathcal{M}}, t_{1}, t_{2}, \gamma\right)(\alpha$, resp.) held fixed.

GZF-BF tailored to proper Gaussian sources for PGS : With the proper Gaussian source $s_{d_{m}}$ having $\mathbb{E}\left(\left|s_{d_{m}}\right|^{2}\right)=1$ in (4) and (6), the information throughput at UE $d_{\ell}$ is $\left(1 / t_{2}\right) \rho_{\ell}(\alpha)$ with $\rho_{\ell}(\alpha)=\ln \left[1+\left|\mathbf{h}_{d_{\ell}} \mathbf{f}_{\ell}\left(\alpha_{\ell}\right)\right|^{2} / L_{\ell}(\alpha)\right]$, where $L_{\ell}(\alpha) \triangleq$ $\sum_{m \in \mathcal{M} \backslash\{\ell\}}\left|\mathbf{h}_{d_{\ell}} \mathbf{f}_{m}\left(\alpha_{m}\right)\right|^{2}+\sigma$. Instead of (9) we now have to deal with the problem

$$
\begin{aligned}
& \max _{\left\{\tilde{p}_{\mathcal{K}}>0, t_{1}, t_{2}, \gamma\right\}>0, \alpha} \text { s.t. } \quad(9 b),(9 d) \\
& \rho_{\ell}(\alpha) \geq 2 \gamma t_{2}, \ell \in \mathcal{M}, \\
& \pi_{1}\left(\tilde{p}_{\mathcal{K}}\right) / t_{1}+\tilde{\pi}_{2}(\alpha) / t_{2} \leq P, \\
& \left\|\mathbf{h}_{e_{j}}\right\|^{2} \tilde{p}_{j} \leq P, j \in \mathcal{K} ; \sum_{i=1}^{\iota_{m}}\left|\alpha_{m, i}\right|^{2} \leq P, m \in \mathcal{M} \text {, }
\end{aligned}
$$

associated with $\tilde{\pi}_{2}(\alpha) \triangleq \sum_{m=1}^{M} \sum_{i=1}^{\iota_{m}}\left|\alpha_{m, i}\right|^{2}$. Let $\left(\tilde{p}_{\mathcal{K}}^{(\kappa)}, \alpha_{\mathcal{M}}^{(\kappa)}, t^{(\kappa)}, t^{(\kappa)}, \gamma^{(\kappa)}\right)$ be the feasible point for $(9)$ found from the $(\kappa-1)$-th iteration. By exploring the inequality (2), we have

$$
\rho_{\ell}(\alpha) \geq \rho_{\ell}^{(\kappa)}(\alpha)
$$

$$
\begin{aligned}
& \text { with the concave function } \rho_{\ell}^{(\kappa)}(\alpha) \triangleq \\
& a_{\ell}^{(\kappa)}+2 \Re\left\{\left(\mathbf{h}_{d_{\ell}} \mathbf{f}_{\ell}\left(\alpha_{\ell}^{(\kappa)}\right)\right)^{*} \mathbf{h}_{d_{\ell}} \mathbf{f}_{\ell}\left(\alpha_{\ell}\right)\right\} / b_{\ell}^{(\kappa)} \quad- \\
& c_{\ell}^{(\kappa)}\left(\left|\mathbf{h}_{d_{\ell}} \mathbf{f}_{\ell}\left(\alpha_{\ell}\right)\right|^{2}+L_{\ell}(\alpha)\right) \text {, for } a_{\ell}^{(\kappa)}=\rho_{\ell}\left(\alpha^{(\kappa)}\right)- \\
& \left|\mathbf{h}_{d_{\ell}} \mathbf{f}_{\ell}\left(\alpha_{\ell}^{(\kappa)}\right)\right|^{2} / b_{\ell}^{(\kappa)}, \quad b_{\ell}^{(\kappa)}=L_{\ell}\left(\alpha^{(\kappa)}\right) \text {, and } c_{\ell}^{(\kappa)}= \\
& \left|\mathbf{h}_{d_{\ell}} \mathbf{f}_{\ell}\left(\alpha_{\ell}^{(\kappa)}\right)\right|^{2} /\left(b^{(\kappa)}\left(b_{\ell}^{(\kappa)}+\left|\mathbf{h}_{d_{\ell}} \mathbf{f}_{\ell}\left(\alpha_{\ell}^{(\kappa)}\right)\right|^{2}\right)\right) \text {. }
\end{aligned}
$$

Algorithm 2, which is based on (19), provides the pseudocode for implementing the proposed path-following computational procedure, which converges at least to a locally optimal solution of (18), similarly to Algorithm 1.

\section{EXPLOITING IGS FOR CONVENTIONAL PS}

Without loss of generality, we assume that the EUs $e_{1}, \ldots, e_{n_{c}}$ and the IUs $d_{1}, \ldots, d_{n_{c}}$ are the same, which constitute the set of simultaneous EUs and IUs. Accordingly, we define $\mathcal{N}_{c} \triangleq\left\{1, \ldots, n_{c}\right\}$. For computational tractability, the PS must use the CBF $\sum_{k \in \mathcal{K} \backslash \mathcal{N}_{c}} \mathbf{h}_{e_{k}}^{H} s_{e_{k}}+\sum_{m \in \mathcal{M}} \mathbf{h}_{d_{m}}^{H} s_{d_{m}}$ [7], but in contrast to [7] both $s_{e_{k}}$ and $s_{d_{m}}$ are assumed to be improper Gaussian, since they will result in the same harvested energy, but the improper Gaussian $s_{e_{k}}$ will impose much weaker interference in ID compared to its proper Gaussian counterpart. Thus, in addition to (7), for $k \in \mathcal{N}_{e} \triangleq \mathcal{K} \backslash \mathcal{N}_{c}$ we define furthermore

$$
\mathbf{C}\left(\tilde{p}_{k}, \tilde{q}_{k}\right)=\left[\begin{array}{cc}
\mathbb{E}\left(\left|s_{e_{k}}\right|^{2}\right) & \mathbb{E}\left(\left(s_{e_{k}}\right)^{2}\right) \\
\mathbb{E}^{*}\left(\left(s_{e_{k}}\right)^{2}\right) & \mathbb{E}\left(\left|s_{e_{k}}\right|^{2}\right)
\end{array}\right]=\left[\begin{array}{cc}
\tilde{p}_{k} & \tilde{q}_{k} \\
\tilde{q}_{k}^{*} & \tilde{p}_{k}
\end{array}\right],
$$

and $\tilde{\mathbf{G}}_{\ell, k}=\operatorname{diag}\left[\left(\mathbf{h}_{d_{\ell}} \mathbf{h}_{e_{k}}^{H}\right) /\left\|\mathbf{h}_{d_{\ell}}\right\|^{2},\left(\mathbf{h}_{d_{\ell}} \mathbf{h}_{e_{k}}^{H}\right)^{*} /\left\|\mathbf{h}_{d_{\ell}}\right\|^{2}\right]$, and $\mathbf{G}_{\ell, m}=\operatorname{diag}\left[\left(\mathbf{h}_{d_{\ell}} \mathbf{h}_{d_{m}}^{H}\right) /\left\|\mathbf{h}_{d_{\ell}}\right\|^{2},\left(\mathbf{h}_{d_{\ell}} \mathbf{h}_{d_{m}}^{H}\right)^{*} /\left\|\mathbf{h}_{d_{\ell}}\right\|^{2}\right]$.

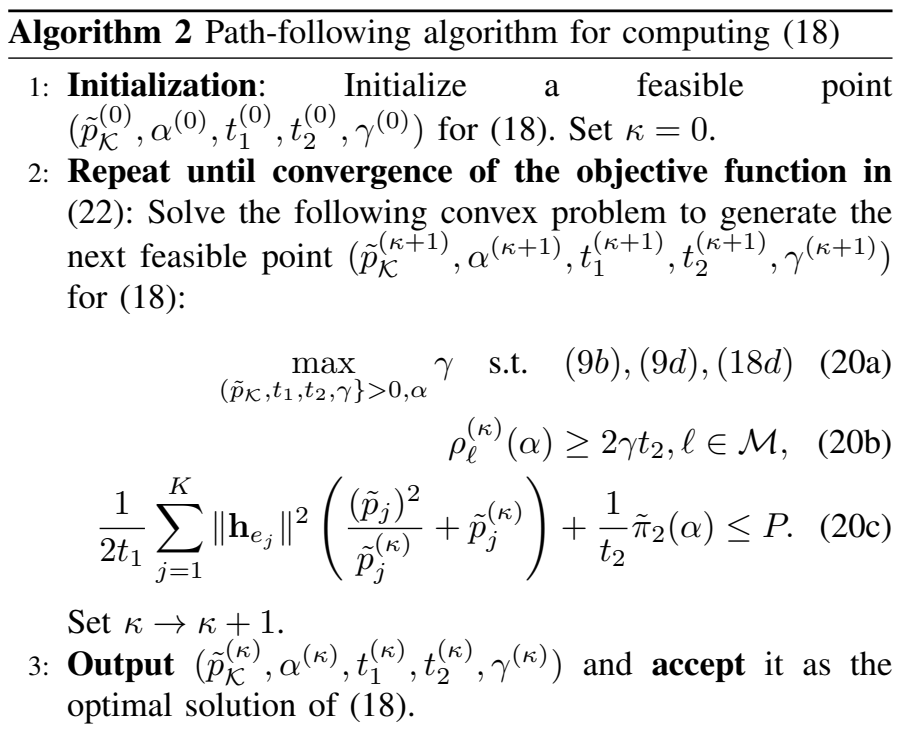

The IU $d_{\ell}$ employs a power splitter to divide its received signal $y_{d_{\ell}}=\mathbf{h}_{d_{\ell}}\left(\sum_{k \in \mathcal{N}_{e}} \mathbf{h}_{e_{k}}^{H} s_{e_{k}}+\sum_{m \in \mathcal{M}} \mathbf{h}_{d_{m}}^{H} s_{d_{m}}\right)+$ $n_{d_{n}}$ during the whole time slot into two parts in the proportion of $0<1 / \alpha_{\ell}<1$. The first part $\sqrt{1 / \alpha_{\ell}} y_{d_{\ell}}$ is processed and used for $\mathrm{EH}$, yielding the harvested energy $\pi_{\ell}^{\mathrm{PS}}\left(\tilde{p}_{\mathcal{N}_{e}}, p_{\mathcal{M}}\right) / \alpha_{\ell}$, where $\pi_{\ell}^{\mathrm{PS}}\left(\tilde{p}_{\mathcal{N}_{e}}, p_{\mathcal{M}}\right) \triangleq$ $\zeta\left(\sum_{k \in \mathcal{N}_{e}} \tilde{p}_{k}\left|\mathbf{h}_{d_{\ell}} \mathbf{h}_{e_{k}}^{H}\right|^{2}+\sum_{m \in \mathcal{M}} p_{m}\left|\mathbf{h}_{d_{\ell}} \mathbf{h}_{d_{m}}^{H}\right|^{2}\right)$. The second part $\sqrt{1 / \beta_{\ell}} y_{d_{\ell}}$ with $1 / \beta_{\ell}=1-1 / \alpha_{\ell}$, is used for ID, yielding the information throughput $(1 / 2) r_{\ell}^{\mathrm{PS}}\left(\tilde{p}_{\mathcal{N}_{e}}, \tilde{q}_{\mathcal{N}_{e}}, p_{\mathcal{M}}, q_{\mathcal{M}}, \beta_{\ell}\right)$, with

$$
\begin{array}{r}
r_{\ell}^{\mathrm{PS}}\left(\tilde{p}_{\mathcal{N}_{e}}, \tilde{q}_{\left.\mathcal{N}_{e}, p_{\mathcal{M}}, q_{\mathcal{M}}, \beta_{\ell}\right)}\right. \\
\ln \left|I_{2}+\mathbf{C}\left(p_{\ell}, q_{\ell}\right) \Lambda_{\ell}^{-1}\left(\tilde{p}_{\mathcal{N}_{e}}, \tilde{q}_{\mathcal{N}_{e}}, p_{\mathcal{M}}, q_{\mathcal{M}}, \beta_{\ell}\right)\right|,
\end{array}
$$

where $\quad \Lambda_{\ell}\left(\tilde{p}_{\mathcal{N}_{e}}, \tilde{q}_{\mathcal{N}_{e}}, p_{\mathcal{M}}, q_{\mathcal{M}}, \beta_{\ell}\right)$

$\sum_{k \in \mathcal{N}_{e}} \tilde{\mathbf{G}}_{\ell, k} \mathbf{C}\left(\tilde{p}_{k}, \tilde{q}_{k}\right) \tilde{\mathbf{G}}_{\ell, k}^{H}$

$\sum_{m \in \mathcal{M} \backslash\{\ell\}} \mathbf{G}_{\ell, m} \mathbf{C}\left(p_{m}, q_{m}\right) \mathbf{G}_{\ell, m}^{H}+\left(\left(\sigma+\sigma_{c} / \beta_{\ell}\right) /\left\|\mathbf{h}_{d_{\ell}}\right\|^{2}\right) \mathbf{I}_{2}$. Here $\sigma_{c}=-90 \mathrm{dBm}$ is the additional noise introduced by the ID receiver circuitry. Note that we have $1 / \alpha_{\ell} \equiv 0$ for $\ell \in \mathcal{M} \backslash \mathcal{N}_{c}$, i.e. there is no PS for ID-only IU. Analogously, there is no PS for EH-only EUs $e_{j}$, $j \in \mathcal{N}_{e}$, whose harvested energy is $\pi_{j}^{\mathrm{PS}}\left(\tilde{p}_{\mathcal{N}_{e}}, p_{\mathcal{M}}\right) \triangleq$ $\zeta\left(\sum_{k \in \mathcal{N}_{e}} \tilde{p}_{k}\left|\mathbf{h}_{e_{j}} \mathbf{h}_{e_{k}}^{H}\right|^{2}+\sum_{m \in \mathcal{M}} p_{m}\left|\mathbf{h}_{e_{j}} \mathbf{h}_{d_{m}}^{H}\right|^{2}\right)$.

Thus, by setting $\alpha_{k} \equiv 1$ for $k \in \mathcal{K} \backslash \mathcal{N}_{c}$ and $1 / \alpha_{m} \equiv 0$ for $m \in \mathcal{M} \backslash \mathcal{N}_{c}$, the problem of max-min throughput optimization subject to EH constraints is formulated as

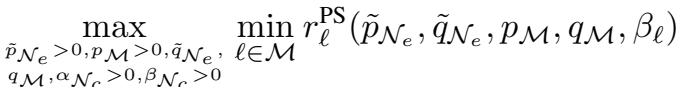

$$
\begin{aligned}
& \text { s.t. } \quad(9 e),\left|\tilde{q}_{k}\right| \leq \tilde{p}_{k}, k \in \mathcal{N}_{e}, \\
& 1 / \alpha_{\ell}+1 / \beta_{\ell} \leq 1, \ell \in \mathcal{N}_{c}, \\
& \pi_{j}^{\mathrm{PS}}\left(\tilde{p}_{\mathcal{N}_{e}}, p_{\mathcal{M}}\right) \geq e_{j}^{\min } \alpha_{j}, j \in \mathcal{K}, \\
& \sum_{j \in \mathcal{N}_{e}}\left\|\mathbf{h}_{e_{j}}\right\|^{2} \tilde{p}_{j}+\sum_{\ell \in \mathcal{M}}\left\|\mathbf{h}_{d_{\ell}}\right\|^{2} p_{\ell} \leq P,
\end{aligned}
$$

which is nonconvex due to the non-concave nature of the objective function (22a).

Let $\left(\tilde{p}_{\mathcal{N}_{e}}^{(\kappa)}, \tilde{q}_{\mathcal{N}_{e}}^{(\kappa)}, p_{\mathcal{M}}^{(\kappa)}, q_{\mathcal{M}}^{(\kappa)}, \alpha_{\mathcal{N}_{c}}^{(\kappa)}, \beta_{\mathcal{N}_{c}}^{(\kappa)}\right)$ be the feasible point for (22) that is found from the $(\kappa-1)$-th iteration. By using the inequality (1), we obtain $r_{\ell}^{\mathrm{PS}}\left(\tilde{p}_{\mathcal{N}_{e}}, \tilde{q}_{\mathcal{N}_{e}}, p_{\mathcal{M}}, q_{\mathcal{M}}, \beta_{\ell}\right) \geq$ 


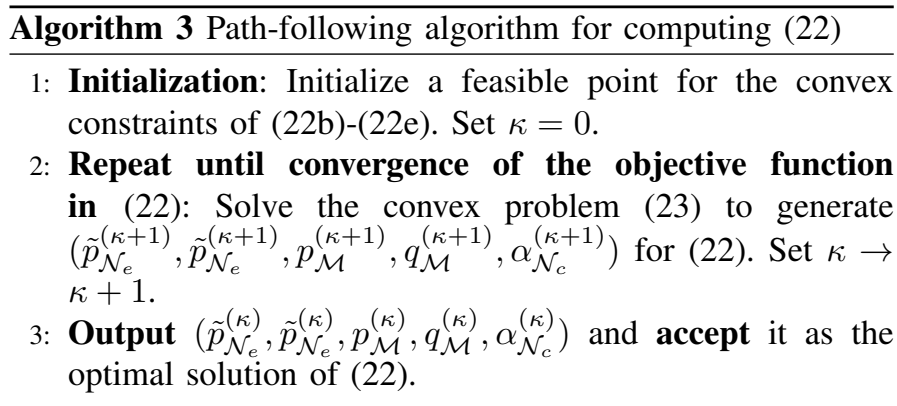

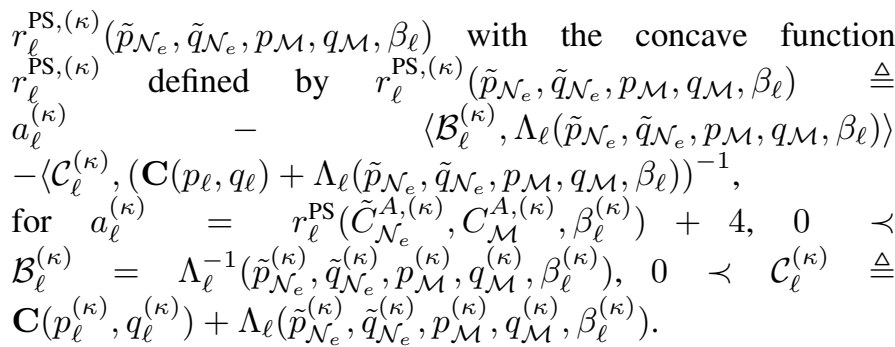

At the $\kappa$ th iteration, the following convex optimization problem can be solved to generate the next iterative feasible point $\left(\tilde{p}_{\mathcal{N}_{e}}^{(\kappa+1)}, \tilde{q}_{\mathcal{N}_{e}}^{(\kappa+1)}, p_{\mathcal{M}}^{(\kappa+1)}, \tilde{q}_{\mathcal{M}}^{(\kappa+1)}, \alpha_{\mathcal{N}_{c}}^{(\kappa+1)}\right)$ for (22):

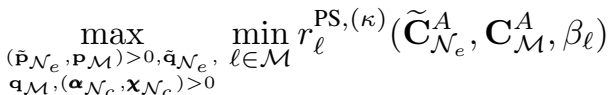

$$
\begin{aligned}
& \text { s.t. }(22 b)-(22 e) \text {. }
\end{aligned}
$$

Algorithm 3 provides the pseudo-code for implementing the proposed path-following computational procedure, which converges at least to a locally optimal solution of (22), similarly to Algorithm 1.

\section{Simulation Results}

In our simulations, the users are randomly placed within a radius of 100 meters from the BS. To ensure meaningful wireless energy harvesting, we place $K=5$ EUs within a radius of 8 meters from the BS. Unless specified otherwise, we assume $M=11$ IUs in our simulations. We consider $n_{c}=3$ overlapping users, who act as both EUs and IUs. Thus we have $\mathcal{K}=\{1,2, \ldots, 5\}, \mathcal{M}=\{1,2, \ldots, M\}$ and $\mathcal{N}_{c}=\{1,2,3\}$. The channel spanning from the BS to a user at a distance of $d$ meters is generated as $\mathbf{h}=\sqrt{10^{-\sigma_{\mathrm{PL}} / 10}} \tilde{\mathbf{h}}$ with the path loss $\sigma_{\mathrm{PL}}=30+10 \beta \log _{10}(d) \mathrm{dB}$ and the normalized channel gain vector $\tilde{\mathbf{h}}$. Here, $\mathbf{h} \equiv \mathbf{h}_{e_{k}}$ if it refers to the channel between the BS and the $k$-th EU or $\mathbf{h} \equiv \mathbf{h}_{d_{\ell}}$ if it refers to the channel between the BS and the $\ell$-th IU and $\beta$ is the path-loss exponent. Generally, the normalized channel vector $\tilde{h}$ follows the Rayleigh distribution with $\beta=3$. However, for the channels between the BS and the nearby users (located within 8-meter radius from the $\mathrm{BS})$, the normalized channel vector $\tilde{\mathbf{h}}$ follows the Rician distribution with Rician factor of $10 \mathrm{~dB}$ and $\beta=2$. We set the energy harvesting threshold as $e_{\min } \geq-20 \mathrm{dBm}$ with $\zeta=0.5$. This is because to enable $\mathrm{EH}$, the power of the signal received at the EUs must exceed the threshold of -21 $\mathrm{dBm}$ (assuming $13 \mathrm{~nm}$ CMOS technology [18]). The bandwidth is set to $B=20 \mathrm{MHz}$, the carrier frequency is set to $2 \mathrm{GHz}$, and the power spectral density of noise is $-174 \mathrm{dBm} / \mathrm{Hz}$. Unless specified otherwise, we assume $N_{t}=10$ antennas at the BS, the transmit power budget of $P=31 \mathrm{dBm}$ and $e_{\min }=-20$ $\mathrm{dBm}$. Thus, the computation for RZF-BF and GZF-BF involves $K+M=16$ real and $M=11$ complex decision variables vs. the number $N_{t}(K+2 M)=270$ of complex decision variables for the computation of beamformers in [11].

In all figures, RZF-BF-IGS represents IGS under transmitside TS approach with RZF-BF (Algorithm 1), GZF-BF-IGS represents IGS under transmit-side TS approach with GZFBF, PS-IGS represents IGS under conventional PS approach, RZF-BF-PGS represents PGS under transmit-side TS approach with RZF-BF, GZF-BF-PGS represents PGS under transmitside TS approach with GZF-BF, and PS-PGS represents PGS under conventional PS approach.

Fig. 1 plots the max-min throughput versus the number of IUs $M$. Fig. 1 shows that the max-min throughput decreases with the increase in the number of IUs because the presence of more users competing for the given fixed resources limits their achievable minimum throughput. We can observe that the IGS based algorithms (RZF-BF-IGS, GZF-BF-IGS and PSIGS) outperform the respective PGS based algorithms (RZFBF-PGS, GZF-BF-PGS and PS-PGS), respectively. Fig. 1 also shows that the transmit-side TS based algorithms (RZFB-IGS, GZFB-IGS, RZFB-PGS, GZFB-PGS) clearly outperform the conventional PS based algorithms (PS-IGS, PGS-PGS), however the performance gap becomes smaller with the increase of $M$.

Fig. 2 and Fig. 3 plot the max-min throughput versus the transmit power budget $\mathrm{P}$ and the energy harvesting threshold $e_{\min }$, respectively. As excepted, the max-min throughput increases upon increasing the available power budget in Fig. 2 due to the availability of more power and decreases with the increase of $e_{\min }$ in Fig. 3, because more resources are required for fulfilling the increased energy harvesting requirement. Upon comparing the performance of RZF-BF and GZF-BF, we can observe that they achieve similar performance under IGS, but GZF-BF slightly outperforms RZF-BF under PGS. Fig. 2 and Fig. 3 also show the supremacy of IGS over PGS and the supremacy of the transmit-TS approach over the conventional PS approach. Note that the surprising gap between the performances of the transmit-TS approach and the conventional PS approach is due to the fact that the former technique exploits the option of distinct energy and information transmit beamformers, while the latter relies on a joint energy and data beamformer.

\section{CONCLUSions}

The paper has developed new regularized zero-forcing beamforming and generalized zero-forcing beamforming for improper Gaussian signaling to serve both energy and information users in an energy-harvesting aided network. They help IGS outperform its proper Gaussian counterpart in terms of the information users' max-min throughput under the same QoS for the energy users. It would be interesting to study their performance both under a nonlinear harvesting model [19] and realistic channel uncertainties [20].

\section{REFERENCES}

[1] J. Hu, K. Yang, G. Wen, and L. Hanzo, "Integrated data and energy communication network: A comprehensive survey," IEEE Commun. Surveys Tuts., vol. 20, no. 4, pp. 3169-3219, Fourthquarter 2018. 


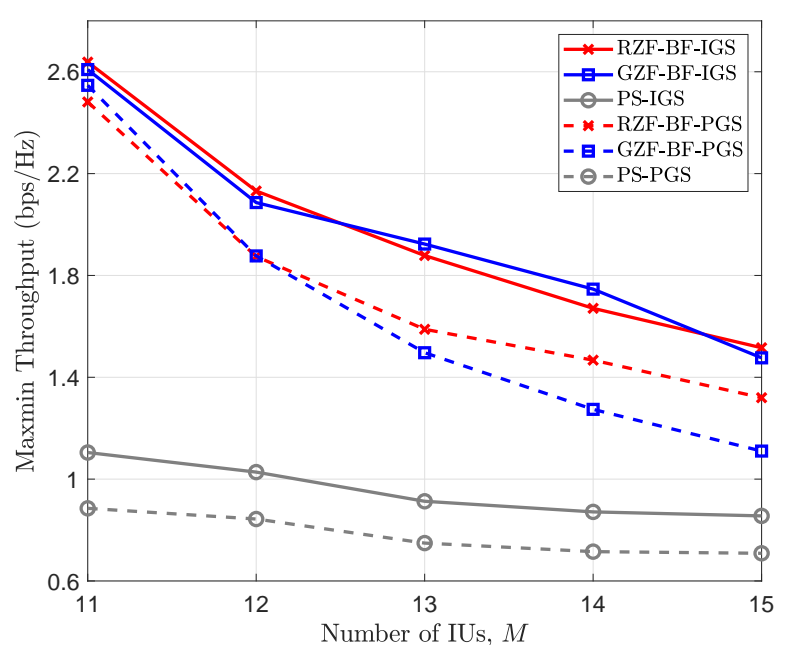

Fig. 1: Max-min throughput versus the number of IUs, $\mathcal{M}$

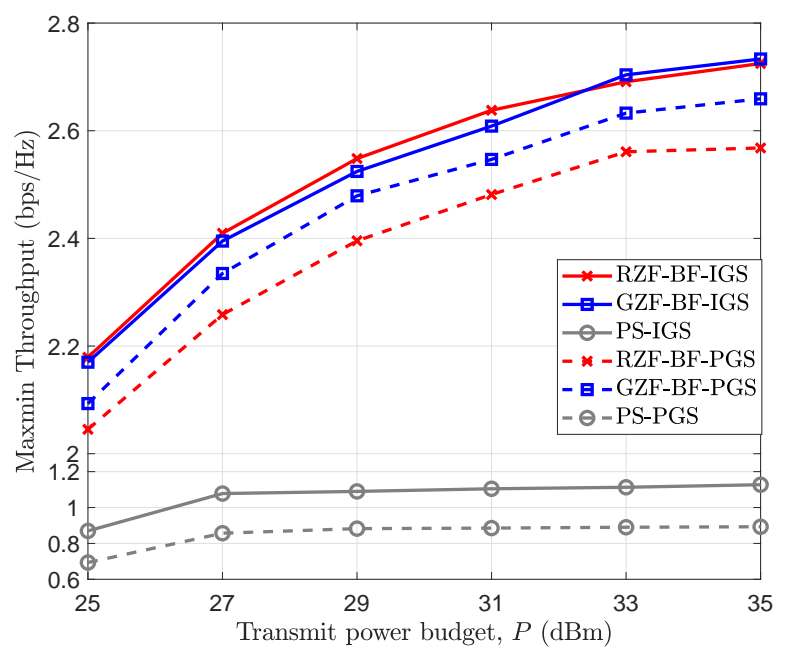

Fig. 2: Max-min throughput versus transmit power budget, $P$ $(\mathrm{dBm})$

[2] K. Xu, Z. Shen, Y. Wang, X. Xia, and D. Zhang, "Hybrid time-switching and power splitting SWIPT for full-duplex massive MIMO systems: A beam-domain approach," IEEE Trans. Veh. Tech., vol. 67, no. 8, pp. 72577274, Aug. 2018.

[3] J. Rostampoor, S. M. Razavizadeh, and I. Lee, "Energy efficient precoding design for SWIPT in MIMO two-way relay networks," IEEE Trans. Veh. Tech., vol. 66, no. 9, pp. 7888-7896, Sept. 2017.

[4] A. A. Nasir, H. D. Tuan, D. T. Ngo, T. Q. Duong, and H. V. Poor, "Beamforming design for wireless information and power transfer systems: Receive power-splitting versus transmit time-switching," IEEE Trans. Commun., vol. 65, no. 2, pp. 876-889, Feb. 2017.

[5] H. H. M. Tam, H. D. Tuan, A. A. Nasir, T. Q. Duong, and H. V. Poor, "MIMO energy harvesting in full-duplex multi-user networks," IEEE Trans. Wirel. Commun., vol. 16, no. 5, pp. 3282-3297, May 2017.

[6] A. A. Nasir, H. D. Tuan, T. Q. Duong, and L. Hanzo, "Transmitter-side time-switching for wireless information and power transfer in massive MIMO systems," IEEE Trans. Veh. Tech., vol. 69, no. 2, pp. 2322-2326, Feb. 2020.

[7] L. Zhao, X. Wang, and K. Zheng, "Downlink hybrid information and energy transfer with massive MIMO," IEEE Trans. Wirel. Commun., vol. 15, no. 2, pp. 1309-1322, Feb. 2016.

[8] C. B. Peel, B. M. Hochwald, and A. L. Swindlerhurst, "A vector-perturbation technique for near capacity multiantenna multiuser communication-part I: channel inversion and regularization," IEEE Trans.

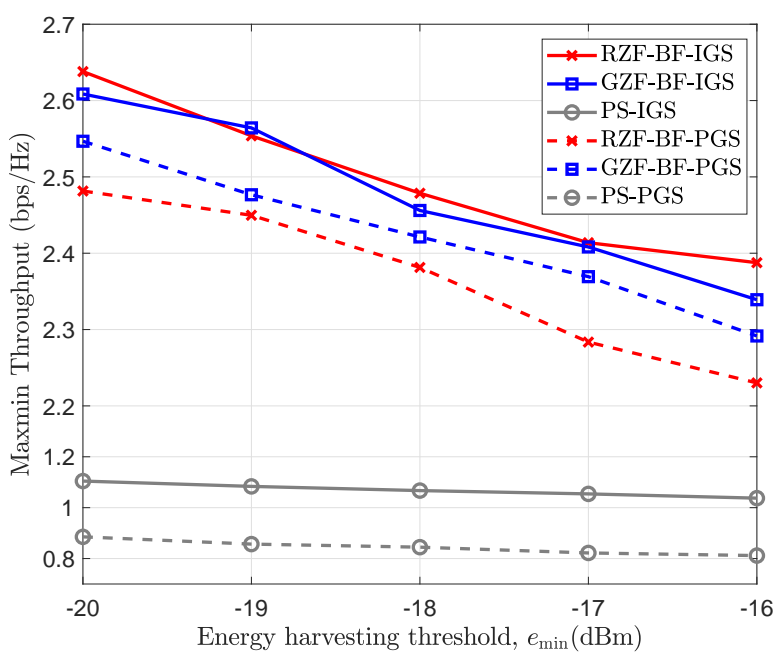

Fig. 3: Max-min throughput versus energy harvesting threshold, $e_{\min }(\mathrm{dBm})$

Commun., vol. 53, no. 1, pp. 195-202, Jan. 2005.

[9] H. T. Nguyen, H. D. Tuan, T. Q. Duong, H. V. Poor, and W.-J. Hwang, "Collaborative multicast beamforming for content delivery by cacheenabled ultra dense networks," IEEE Trans. Commun., vol. 67, no. 5, pp. 3396-3406, May 2019.

[10] I. E. Telatar, "Capacity of multi-antenna Gaussian channels," Eur. Trans. Telecommun., vol. 10, no. 6, pp. 585-595, Nov./Dec. 1999.

[11] H. Yu, H. D. Tuan, T. Q. Duong, Y. Fang, and L. Hanzo, "Improper Gaussian signaling for integrated data and energy networking," IEEE Trans. Commun. (early access), 2020.

[12] P. J. Schrerier and L. L. Scharf, Statistical Signal Processing of ComplexValued Data: The Theory of Improper and Noncircular Signals. Cambridge University Press, 2010.

[13] S. Javed, O. Amin, B. Shihada, and M.-S. Alouini, "A journey from improper Gaussian signaling to asymmetric signaling," IEEE Commun. Surv. Tut.(Early Access), 2020.

[14] L. D. Nguyen, H. D. Tuan, T. Q. Duong, and H. V. Poor, "Multiuser regularized zero-forcing beamforming," IEEE Trans. Signal Process., vol. 67, no. 11, pp. 2839-2853, Jun. 2019.

[15] H. H. M. Tam, H. D. Tuan, and D. T. Ngo, "Successive convex quadratic programming for quality-of-service management in full-duplex MUMIMO multicell networks," IEEE Trans. Commun., vol. 64, no. 6, pp. 2340-2353, June 2016.

[16] T. M. Cover and J. A. Thomas, Elements of Information Theory (second edition). John Wileys \& Sons, 2006.

[17] H. Tuy, Convex Analysis and Global Optimization (second edition). Springer International, 2016.

[18] X. Lu, P. Wang, D. Niyato, D. I. Kim, and Z. Han, "Wireless networks with RF energy harvesting: A contemporary survey," IEEE Commun. Surveys Tuts., vol. 17, pp. 757-789, 2015.

[19] M. Varasteh, B. Rassouli, and B. Clerckx, "Wireless information and power transfer over an AWGN channel: Nonlinearity and asymmetric Gaussian signaling," in Proc. 2017 IEEE Info. Theory Works (ITW), 2017, pp. 181-185.

[20] S. Abeywickrama, T. Samarasinghe, C. K. Ho, and C. Yuen, "Wireless energy beamforming using received signal strength indicator feedback," IEEE Trans. Signal Process., vol. 66, no. 1, pp. 224-235, Jan. 2018. 